\title{
Fuzzy pre-continuous and fuzzy pre*-continuous function of fuzzy pre-compact space in fuzzy topological space
}

\author{
Munir Abdul Khalik Al-Khafaji*, Marwah Flayyih Hasan \\ Department of Mathematics, College of Education, Al-Mustansiriya University, Baghdad, Iraq \\ *Corresponding author E-mail: mnraziz@yahoo.com
}

Copyright $\odot 2015$ Munir Abdul Khalik Al-Khafaji, Marwah Flayyih Hasan. This is an open access article distributed under the Creative Commons Attribution License, which permits unrestricted use, distribution, and reproduction in any medium, provided the original work is properly cited.

\begin{abstract}
The aim of this paper is to introduce and study the notion of a fuzzy pre-continuous function, fuzzy pre*- continuous function, fuzzy pre-compact space and some properties, remarks related to them.

Keywords: Fuzzy Pre-Continuous.Fuzzy Pre*-Continuous; Fuzzy Compact Space in Fuzzy Topological Space.

\section{Introduction}

The concept of fuzzy set was introduced by Zadeh (1965) in classical paper. Chang (1968) introduced the notion of a fuzzy topology. Also in (1992) Chakraborty M.K and T.M.G Ahsanullah Introduction fuzzy topology on fuzzy sets in (1993) Chauldhuri M.K and P.Das Introduced some results on fuzzy topology on fuzzy set.

In (1997) Ganesan Balasubamanian introduction on fuzzy $\beta$-compact and fuzzy $\beta$ - extermally disconnected space Also in (2004) I.M.Hanafy Introduced fuzzy $\beta$ - compactness and fuzzy $\beta$-closed space. Also, in (2007) M.K.uma, EROJA and G.BALASUBAL MANINA Introduced on fuzzy $\beta$-compact * spaces and fuzzy filters in (2009) Atallah Th.AL-Ani Introduced Z-compact space. Also in (2011) J.Karnel introduced fuzzy, b-compact and fuzzy b-closed spaces.
\end{abstract}

\section{Fuzzy pre-compact space}

In this section we study some definitions, remarks, Propositions and theorems about fuzzy Pre- compact space in fuzzy topological spaces.

Definition 2.1: [1] [2] A collection $\widetilde{T}$ of a sub set of $\widetilde{A}$ that is $\widetilde{T} \subseteq p(\widetilde{A})$ is said to be fuzzy topology on $\widetilde{A}$ if satisfies the following condition:

1) $\widetilde{\emptyset}, \widetilde{\mathrm{A}} \in \widetilde{\mathrm{T}}$

2) If $\widetilde{G}, \widetilde{H} \in \widetilde{T}$, Then $\widetilde{G} \cap \widetilde{H} \in \widetilde{T}$

3) If $\widetilde{\mathrm{G}} \mathrm{i} \in \widetilde{\mathrm{T}}$, Then $\cup \widetilde{\mathrm{G}} \mathrm{i} i \in \lambda$

The pair $(\widetilde{A}, \widetilde{T})$ is said to be fuzzy topological, every member of $\widetilde{\mathrm{T}}$ is called fuzzy open ( $\widetilde{\mathrm{T}}$-open) set in $\widetilde{\mathrm{A}}$ and the complement is called fuzzy ( $\widetilde{\mathrm{T}}-$ closed) set .

Definition 2.2: [8] Let $(\widetilde{A}, \widetilde{T})$ be a fuzzy topological spaces a family W of fuzzy sets is pre - open cover of a fuzzy set $\widetilde{B}$ if and only if $\widetilde{B} \subseteq U\{\widetilde{C}: \widetilde{C} \in \widetilde{W}\}$ and each member of $W$ is a fuzzy pre- open set. A sub cover of $W$ is a sub family which is also cover.

Definition 2.3: [8] A fuzzy topological spaces $(\widetilde{\mathrm{A}}, \widetilde{T})$ is fuzzy pre- compact if and only if every fuzzy pre- open cover of $\widetilde{\mathrm{A}}$ has a finite sub cover. 
Definition 2.4: [4] Let $\widetilde{B}$ is fuzzy sub set of a fuzzy topological space $(\widetilde{\mathrm{A}}, \widetilde{\mathrm{T}}), \widetilde{B}$ is said to be fuzzy pre-open relative to $\widetilde{\mathrm{A}}$ if for every fuzzy pre- open cover $\left\{\widetilde{\mathrm{N}}_{\lambda}: \lambda \in \Lambda\right\}$ such that $\widetilde{\mathrm{N}}_{\lambda}$ is fuzzy pre-open sets in $(\widetilde{\mathrm{A}}, \widetilde{\mathrm{T}})$ having finite sub cover.

Definition 2.5: [10] A fuzzy topological space $(\widetilde{\mathrm{A}}, \widetilde{T})$ is said to be fuzzy (pre- $\widetilde{T}_{2}$ ) i.e. (fuzzy pre- Housdorff) if for each pair of distinct point $\tilde{x}, \tilde{y}$ of $(\widetilde{A}, \widetilde{T})$, there exists disjoint fuzzy pre-open sets $\widetilde{U}$ and $\widetilde{V}$ such that $\tilde{x} \in \widetilde{U}$ and $\tilde{y} \in \widetilde{V}$

Remark 2.6: [8] Every fuzzy open cover is a fuzzy pre-open cover.

Proposition 2.7: [4] Every fuzzy pre-compact is fuzzy compact space.

Proof:

Let $(\widetilde{\mathrm{A}}, \widetilde{\mathrm{T}})$ is fuzzy pre-compact space

And $\left\{\widetilde{\mathrm{N}}_{\lambda}: \lambda \in \boldsymbol{\Lambda}\right\}$ is open cover to $\widetilde{\mathrm{A}}$

$\therefore\left\{\widetilde{\mathrm{N}}_{\lambda}: \lambda \in \boldsymbol{\Lambda}\right\}$ is pre-open cover to $\widetilde{\mathrm{A}}$

$\because \widetilde{\mathrm{A}}$ is fuzzy pre-compact space.

Such that $\mu_{\widetilde{\mathrm{A}}}(\mathrm{x})=\max \left\{\mu_{\widetilde{\mathrm{N}}_{\lambda \mathrm{i}}}(\mathrm{x}): \boldsymbol{\lambda} \in \boldsymbol{\Lambda}\right\}, \mathrm{i}=1,2,3 \ldots \mathrm{n}$

$\therefore \widetilde{\mathrm{A}}$ is fuzzy compact space

Remark 2.8: [4] Fuzzy compact space need not to be fuzzy pre-compact space Proposition (2.9) [7]

If every fuzzy pre-open subset of a fuzzy topological space $(\widetilde{\mathrm{A}}, \widetilde{\mathrm{T}})$ is a fuzzy pre-compact, then every subset of $\widetilde{\mathrm{A}}$ is a fuzzy pre-compact.

Proof:

Obvious

Proposition (2.10) [4]

A fuzzy pre-closed subset of fuzzy pre-compact space is fuzzy pre-compact

Proof:

Suppose that $(\widetilde{\mathrm{A}}, \widetilde{\mathrm{T}})$ be a fuzzy pre-compact space

And $\widetilde{B}$ be a fuzzy pre-closed subset of $(\widetilde{\mathrm{A}}, \widetilde{\mathrm{T}})$

And $\left\{\mu_{\widetilde{N}_{\lambda}}(\mathrm{x}): \lambda \in \boldsymbol{\Lambda}\right\}$ is pre-open cover to $\widetilde{\mathrm{B}}$

Since $\widetilde{\mathrm{B}}^{\mathrm{c}}$ is a fuzzy pre-open cover.

Such that $\max \left\{\left\{\mu_{\widetilde{N}_{\lambda}}(\mathrm{x}): \lambda \in \boldsymbol{\Lambda}\right\}, \mu_{\widetilde{\mathrm{B}}^{\mathrm{c}}}(\mathrm{x})\right\}$ is fuzzy pre-open cover of $\widetilde{\mathrm{B}}$.

Since $(\widetilde{\mathrm{A}}, \widetilde{\mathrm{T}})$ is fuzzy pre-compact space.

Hence $\widetilde{B}$ is fuzzy pre-compact space

Corollary (2.11) [4]

A fuzzy closed subset of a fuzzy pre-compact is fuzzy compact.

Proof:

Suppose that $\mu_{\widetilde{\mathrm{C}}}(\mathrm{x})=\max \left\{\mu_{\widetilde{\mathrm{N}}_{\lambda}}(\mathrm{x}): \lambda \in \boldsymbol{\Lambda}\right\}$ be fuzzy pre-open cover to $\widetilde{\mathrm{B}}$

Suppose that $\mu_{\widetilde{\mathrm{C}}_{1}}(\mathrm{x})=\max \left\{\mu_{\widetilde{\mathrm{C}}_{1}}(\mathrm{x}) \mu_{\widetilde{\mathrm{B}}^{\mathrm{c}}}(\mathrm{x})\right\}$

$\therefore \widetilde{\mathrm{C}}_{1}$ is fuzzy open cover to $(\widetilde{\mathrm{A}}, \widetilde{\mathrm{T}})$

$\because \widetilde{\mathrm{A}}$ is a fuzzy pre-compact.

Such that $\mu_{\widetilde{\mathrm{A}}}(\mathrm{x}) \leq \max \left\{\max \left(\mu_{\widetilde{\mathrm{N}}_{\lambda}}(\mathrm{x}), \mu_{\widetilde{\mathrm{B}}^{c}}(\mathrm{x})\right\}\right.$

$\therefore \mu_{\widetilde{\mathrm{B}}}(\mathrm{x}) \leq \max \left\{\mu_{\widetilde{N}_{\lambda}}(\mathrm{x})\right\}$

Then $\widetilde{B}$ is fuzzy pre- compact

Remark 2.12: [6] A fuzzy pre- closed subset of a fuzzy compact space is needed not to be fuzzy compact.

Theorem 2.13: [8] Let $(\tilde{A}, \tilde{T})$ be a fuzzy topological space if $\tilde{B}$ and $\tilde{C}$ are two Fuzzy pre-compact subsets of $\tilde{A}$, then $\tilde{B} \cup \tilde{C}$ is also fuzzy pre- compact.

Proof :

Suppose that $\left\{\widetilde{\mathrm{N}}_{\lambda}: \lambda \in \boldsymbol{\Lambda}\right\}$ be a fuzzy pre- open cover of $\widetilde{\mathrm{B}} \cup \tilde{\mathrm{C}}$

Then $\max \left\{\mu_{\widetilde{\mathrm{B}}}(\mathrm{x}), \mu_{\widetilde{\mathrm{C}}}(\mathrm{x})\right\} \leq \max \left\{\mu_{\widetilde{\mathrm{N}}_{\lambda}}(\mathrm{x}): \lambda \in \boldsymbol{\Lambda}\right\}$

Since $\mu_{\widetilde{\mathrm{B}}}(\mathrm{x}) \leq \max \left\{\mu_{\widetilde{\mathrm{B}}}(\mathrm{x}), \mu_{\widetilde{\mathrm{C}}}(\mathrm{x})\right\}$

And $\mu_{\widetilde{\widetilde{C}}}(\mathrm{x}) \leq \max \left\{\mu_{\widetilde{\mathrm{B}}}(\mathrm{x}), \mu_{\widetilde{\mathrm{C}}}(\mathrm{x})\right\}$

Also $\left\{\widetilde{\mathrm{N}}_{\lambda}: \lambda \in \boldsymbol{\Lambda}\right\}$ is fuzzy pre- open cover of $\widetilde{\mathrm{B}}$ and a fuzzy pre- open cover of $\widetilde{\mathrm{C}}$ 
Since, $\widetilde{\mathrm{B}}$ and $\widetilde{\mathrm{C}}$ are two pre- compact sets, then there exists a finite sub cover

$\left(, \widetilde{\mathrm{N}}_{\lambda 1}, \widetilde{\mathrm{N}}_{\lambda 2}, \ldots \ldots . \widetilde{\mathrm{N}}_{\lambda \mathrm{m}}\right)$ and $\mu_{\widetilde{\mathrm{B}}}(\mathrm{x}) \leq \max \left\{\mu_{\widetilde{\mathrm{N}}_{\lambda \mathrm{i}}}(\mathrm{x})\right\}, \mathrm{i}=1,2,3, \ldots . \mathrm{n}$

Hence $\mu_{\widetilde{\mathrm{C}}}(\mathrm{x}) \leq \max \left\{\mu_{\widetilde{N}_{\lambda \mathrm{i}}}(\mathrm{x})\right\}$

And $\max \left\{\mu_{\widetilde{\mathrm{B}}}(\mathrm{x}), \mu_{\widetilde{\mathrm{C}}}(\mathrm{x})\right\} \leq \max \left\{\mu_{\widetilde{\mathrm{N}}_{\lambda \mathrm{k}}}(\mathrm{x})\right\}, \mathrm{k}=1,2,3 \ldots \mathrm{n}+\mathrm{m}$

Thus, $\widetilde{\mathrm{A}} \cup \widetilde{\mathrm{B}}$ is fuzzy pre compact

Remark 2.14: [8] If $\widetilde{B}$ and $\widetilde{C}$ are a fuzzy pre-compact subsets of a fuzzy topological space $(\widetilde{A}, \widetilde{T})$ then $\widetilde{B} \cap \widetilde{C}$ is need not to be fuzzy pre-compact space.

Theorem 2.15: [4] Every fuzzy pre-closed off $(\tilde{A}, \tilde{T})$ is fuzzy pre-compact if and only if $(\tilde{A}, \tilde{T})$ is fuzzy pre-compact.

Proof:

Let $\left\{\widetilde{\mathrm{N}}_{\lambda}: \lambda \in \boldsymbol{\Lambda}\right\}$ is fuzzy pre-open cover in $\widetilde{\mathrm{A}}$

Then $\mu_{\widetilde{\mathrm{A}}}(\mathrm{x})=\max \left\{\mu_{\widetilde{\mathrm{N}}_{\lambda}}(\mathrm{x})^{\mathrm{c}}(\mathrm{x}): \lambda \in\right\}$

Suppose that $\mu_{\widetilde{\mathrm{K}}}(\mathrm{x})=\max \left\{\mu_{\widetilde{\mathrm{N}}_{\lambda}}(\mathrm{x})^{\mathrm{c}}(\mathrm{x}): \lambda \in\right\}$

Then $\widetilde{\mathrm{K}}$ is fuzzy closed.

And $\mu_{\widetilde{\mathrm{K}}}(\mathrm{x}) \leq \max \left\{\mu_{\widetilde{\mathrm{N}}_{\lambda}}(\mathrm{x}): \boldsymbol{\lambda} \in \boldsymbol{\Lambda}-\left\{\lambda_{0}\right\}\right\}$

Then there exist $\Lambda^{-}$fuzzy subset finite on $\Lambda-\left\{\lambda_{0}\right\}$

Such that $\mu_{\widetilde{\mathrm{K}}}(\mathrm{x}) \leq \max \left\{\mu_{\widetilde{\mathrm{N}}_{\lambda}}(\mathrm{x}): \lambda \in \Lambda^{-}\right\}$

Then $\mu_{\widetilde{\mathrm{B}}}(\mathrm{x})=\max \left\{\mu_{\widetilde{\mathrm{K}}}(\mathrm{x}), \mu_{\widetilde{\mathrm{N}}_{\lambda}}(\mathrm{x})\right\}$

And $\mu_{\widetilde{B}}(\mathrm{x}) \leq \max \left\{\mu_{\widetilde{N}_{\lambda}}(\mathrm{x}): \lambda \in \boldsymbol{\Lambda}-\left\{\lambda_{0}\right\}\right\}$

Then $\widetilde{A}$ is fuzzy pre-compact.

Conversely $\leftarrow$

Let $\tilde{B}$ is fuzzy pre-compact

Suppose that $\widetilde{K}$ is fuzzy closed set in $\tilde{A}$

Then $\widetilde{K}$ is fuzzy pre-compact

Theorem 2.16: [3] A fuzzy topological space $(\tilde{A}, \tilde{T})$ is fuzzy pre-compact If and only if for every collection $\left\{\widetilde{N}_{\lambda}: \lambda \in \boldsymbol{\Lambda}\right\}$ of fuzzy pre-closed set of $(\tilde{A}, \tilde{T})$ having the finite intersection property

$\operatorname{Min}\left\{\mu_{\widetilde{N}_{\lambda i}}(\mathrm{x})\right\} \neq \mu_{\widetilde{\varnothing}}(\mathrm{x})$

Proof:

Suppose that $\left\{\widetilde{N}_{\lambda}: \lambda \in\right\}$ be a collection of fuzzy pre- closed with the finite intersection property

Let $\min \left\{\mu_{\widetilde{N}_{\lambda}}(\mathrm{x})\right\}=\mu_{\widetilde{\emptyset}}(\mathrm{x})$ and $\max \left\{\mu_{\widetilde{N}_{\lambda}}(\mathrm{x})\right\}=\mu_{\tilde{A}}(\mathrm{x})$

Since $=\left\{\widetilde{N}_{\lambda}: \lambda \in \boldsymbol{\Lambda}\right\}$ is a collection of fuzzy pre- open set cover of $\tilde{A}$ it follows that there exists a finite subset $\mu \subset \boldsymbol{\Lambda}$

Such that $\max \left\{\mu_{\widetilde{N}_{\lambda}}(\mathrm{x})=\mu_{\tilde{A}}(\mathrm{x})\right.$

Then $\min \left\{\mu_{\widetilde{N}_{\lambda}}(\mathrm{x})\right\}=\mu_{\widetilde{\varnothing}}(\mathrm{x})$ where $\boldsymbol{\Lambda} \in \mu$

Which the contradiction

And therefore $\min \left\{\mu_{\widetilde{N}_{\lambda i}}(\mathrm{x})\right\} \neq \mu_{\widetilde{\emptyset}}(\mathrm{x})$

Conversely

Obvious

Theorem 2.16: [4] Let $\tilde{B}$ is fuzzy open subset of $(\tilde{A}, \tilde{T})$, then $\tilde{B}$ is fuzzy pre-compact if and only if $\tilde{B}$ sub space to $\tilde{A}$.

Proof:

Suppose that $\tilde{B}$ is fuzzy pre-compact to $\tilde{A}$

Suppose that $\left\{\widetilde{N}_{\lambda}(\mathrm{x}): \lambda \in \boldsymbol{\Lambda}\right\}$ is covering to $\tilde{B}$

Such that $\widetilde{N}_{\lambda}$ is fuzzy pre- open set in $\tilde{A}$

Thus $\min \left\{\mu_{\tilde{B}}(\mathrm{x}), \mu_{\widetilde{N}_{\lambda}}(\mathrm{x})\right\}$ is fuzzy pre- open set in $\tilde{A}$

Then $\left\{\widetilde{N}_{\lambda}(\mathrm{x}): \lambda \in\right\}$ is fuzzy pre- open cover to $\widetilde{B}$

$\because \tilde{B}$ is fuzzy pre-compact

Then $\mu_{\tilde{B}}(\mathrm{x})<\max \left\{\min \left\{\mu_{\tilde{B}}(\mathrm{x}), \mu_{\widetilde{N}_{\lambda}}(\mathrm{x}): \lambda \in \Lambda^{-}\right\}\right.$such that $\Lambda^{-} \subset \boldsymbol{\Lambda}$

And $\mu_{\tilde{B}}(\mathrm{x})<\max \left\{\mu_{\widetilde{N}_{\lambda}}(\mathrm{x}): \lambda \in \Lambda^{-}\right\}$ 
$\therefore \tilde{B}$ is fuzzy pre- compact sub space to $\tilde{A}$

Theorem 2.17: [4] Every fuzzy pre- compact space in fuzzy Housdorff space is fuzzy pre-closed

Proof:

Suppose that $(\tilde{B}, \widetilde{T})$ is fuzzy pre- compact in fuzzy Housdorff space.

$\therefore \tilde{B}$ is fuzzy compact space

$\because \tilde{A}$ is fuzzy Housdorff space

$\therefore \tilde{B}$ is fuzzy closed

$\therefore \tilde{B}$ is fuzzy pre- closed in $\tilde{A}$

Proposition 2.18: [4] Let $\tilde{B}, \tilde{C}$ be two fuzzy subset of $(\tilde{A}, \tilde{T}), \tilde{B} \subset \tilde{C}$ and $\tilde{C}$ is fuzzy open set of $\tilde{A}$, then $\tilde{B}$ is fuzzy precompact relative to subspace $\tilde{C}$ if and only if $\tilde{B}$ is fuzzy pre- compact relative to $\tilde{A}$.

Proof:

Suppose that $\tilde{B}$ is fuzzy pre-compact subspace in $\tilde{C}$

$\therefore \tilde{B}$ is fuzzy pre-compact relative to $\tilde{C}$

And $\tilde{B}$ is fuzzy pre-compact relative to $\tilde{A}$

Hence $\tilde{B}$ is fuzzy pre-compact in $\tilde{A}$

Conversely

Let $\tilde{B}$ is fuzzy pre-compact in $\tilde{A}$

$\therefore \widetilde{B}$ is fuzzy pre-compact relative to $\tilde{A}$

Then $\tilde{B}$ is fuzzy pre-compact relative to $\tilde{C}$

Hence $\tilde{B}$ is fuzzy pre-compact in $\tilde{C}$

\section{Fuzzy pre- continuous and fuzzy pre*-continuous}

Definition 3.1: [3] A function f: $(\tilde{A}, \tilde{T}) \rightarrow\left(\widetilde{B}, \tilde{T}^{\prime}\right)$ is fuzzy continuous (f- continuous) if and only if the inverse image of any fuzzy open set in $\tilde{T}^{\prime}$ is fuzzy open set in $\tilde{T}$.

Definition 3.2: A function f: $(\tilde{A}, \widetilde{T}) \rightarrow\left(\widetilde{B}, \tilde{T}^{\prime}\right)$ is said to be a fuzzy pre- continuous if and only if the invers image of any fuzzy open set in $\widetilde{T}$ is fuzzy pre-open set in $\widetilde{T}$.

Definition 3.3: A function $\mathrm{f}:(\tilde{A}, \widetilde{T}) \rightarrow\left(\widetilde{B}, \widetilde{T}^{\prime}\right)$ is said to be a fuzzy pre*- continuous if and only if the invers image of any fuzzy pre- open set in $\tilde{T}^{\prime}$ is fuzzy pre-open set in $\tilde{T}$.

Proposition 3.5: [3] If $\mathrm{f}:(\tilde{A}, \tilde{T}) \rightarrow\left(\tilde{B}, \tilde{T}^{\prime}\right)$ is fuzzy continuous function, then $\mathrm{f}$ is fuzzy pre*-continuous

Proof:

Suppose that $(\tilde{C})$ is a fuzzy pre-open fuzzy in $\tilde{B}$

Then $\mu_{(\tilde{C})}(\mathrm{x}) \leq \mu_{i n t(c l(\tilde{C})}(\mathrm{x})$ and $\mu_{f^{-1}(\tilde{C})}(\mathrm{x}) \leq \mu_{f^{-1}}{ }_{\operatorname{int}(c l(\tilde{C})}(\mathrm{x}) \leq \mu_{i n t\left(f^{-1}(c l(\tilde{C})\right.}(\mathrm{x})$

Since $\mathrm{f}$ is fuzzy continuous

Then $\mu_{f^{-1}{ }_{i n t}(c l(\tilde{C})}(\mathrm{x}) \leq \mu_{i n t\left(f^{-1}\right.}{ }_{c l(\tilde{C})}(\mathrm{x}) \leq \mu_{\operatorname{int}\left(c l\left(f^{-1}(\tilde{C})\right.\right.}(\mathrm{x})$

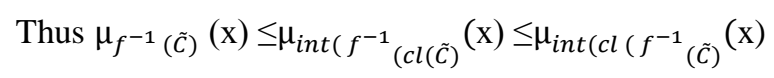

Then $\mathrm{f}$ is fuzzy pre* - continuous

Theorem 3.6: [3] Let $\left.f:(\tilde{A}, \widetilde{T}) \rightarrow(\tilde{B}, \widetilde{T})^{\prime}\right)$ be a function, and then the following are equivalent l-f is fuzzy pre*-continuous $2-f(p-c l(\tilde{C}) \subseteq p(c l(f(\tilde{C}))$, for every fuzzy set $\tilde{C}$ in $\tilde{A}$

Proof:

$2 \rightarrow 1$ Suppose that $\tilde{C}$ be a fuzzy set of $\tilde{A}$

Then p-cl-(f $(\tilde{C})$ is fuzzy pre-closed 
By the (1) $f^{-1}(\mathrm{p}-\mathrm{cl}(\mathrm{f}(\tilde{C})$ is pre-closed

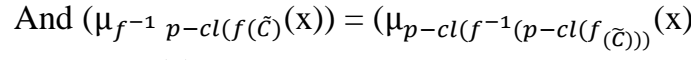

Since $\mu_{(\tilde{C})}(\mathrm{x}) \leq \mu_{f\left(f^{-1}(\tilde{C})\right)}(\mathrm{x})$

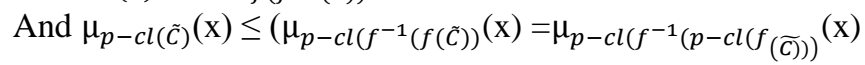

Hence $\mu_{f(p-c l(\tilde{C})(}(\mathrm{x}) \leq \mu_{p-c l\left(f_{(\widetilde{C})}\right.}(\mathrm{x})$

$2 \leftarrow 1$ suppose that $\widetilde{D}$ be a fuzzy pre-closed set in $\widetilde{B}$

And If $\mu_{(\tilde{C})}(\mathrm{x})=\mu_{f^{-1}(\widetilde{D})}(\mathrm{x})$

Then $\mu_{p-c l\left(f^{-1}(\widetilde{D})\right.}(\mathrm{x}) \leq \mu_{f^{-1}\left(p-c l\left(f\left(f^{-1}(\widetilde{D})\right)\right)\right.}(\mathrm{x})$

$\leq \mu_{f^{-1}(p-c l(\widetilde{D}))}(\mathrm{x})=\mu_{f^{-1}(\widetilde{D})}(\mathrm{x})$

Since $\mu_{f^{-1}(\widetilde{D})}(\mathrm{x}) \leq \mu_{p-c l\left(f^{-1}(\widetilde{D})\right.}(\mathrm{x})$

Then $\mu_{f^{-1}(\widetilde{D})}(\mathrm{x})=\mu_{p-c l\left(f^{-1}(\widetilde{D})\right.}(\mathrm{x})$

Hence $f^{-1}(\widetilde{D})$ is fuzzy pre-closed set in $\widetilde{B}$ and $\mathrm{f}$ is fuzzy pre*-continuous

Corollary 3.7: [3] Let f: $(\tilde{A}, \tilde{T}) \rightarrow\left(\widetilde{B}, \widetilde{T}^{\prime}\right)$ be a function, then the following are equivalent

1) $\mathrm{f}$ is fuzzy pre-continuous

2) $\mathrm{f}(\mathrm{p}-\mathrm{cl}(\tilde{C}) \subseteq \operatorname{cl}-(\mathrm{f}(\tilde{C}))$, for every fuzzy set $\tilde{C}$ in $\tilde{A}$

Proof:

Obvious

Proposition 3.8: [3] If $\mathrm{f}:(\tilde{A}, \tilde{T}) \rightarrow\left(\tilde{B}, \tilde{T}^{\prime}\right)$ is fuzzy open and fuzzy continuous function and $\tilde{A}$ is a fuzzy pre- compact Then $\mathrm{f}(\tilde{A})$ is fuzzy pre- compact space.

Proof:

Obvious

Theorem 3.9: [8] The fuzzy pre-continuous image of a fuzzy pre-compact space is fuzzy compact space.

Proof:

Suppose that $(\tilde{A}, \tilde{T})$ be a fuzzy pre- compact space

And f: $(\tilde{A}, \widetilde{T}) \rightarrow\left(\widetilde{B}, \widetilde{T}^{\prime}\right)$ be a fuzzy pre continuous function

To prove $\left(\widetilde{B}, \widetilde{T}^{\prime}\right)$ is a fuzzy compact space

Let $\left\{\widetilde{N}_{\lambda}: \lambda \in \boldsymbol{\Lambda}\right\}$ is a fuzzy open cover of $\widetilde{B}$.

Then $\left\{f^{-1} \widetilde{N}_{\lambda}(\mathrm{x}): \boldsymbol{\lambda} \in \boldsymbol{\Lambda}\right\}$ is fuzzy pre-open cover of $\tilde{A}$

Since $\mathrm{f}$ is fuzzy pre - continuous function a finite sub cover $\left\{\mu_{f^{-1}\left(\widetilde{N}_{\lambda i}\right)}(\mathrm{x}) \mathrm{i}=1,2,3 \ldots \mathrm{n}\right\}$ which covering $\tilde{A}$

Then $\widetilde{B}$ is a fuzzy compact space

Remark 3.10: [4] The fuzzy continuous image of fuzzy pre-compact need not be a fuzzy pre-compact space.

Theorem 3.11: [3] If a function $f:(\tilde{A}, \widetilde{T}) \rightarrow(\widetilde{B}, \widetilde{T})$ is fuzzy pre ${ }^{*}$ - continuous and $\tilde{C}$ is a fuzzy pre-compact relative to $\tilde{A}$ then so is $f(\tilde{C})$ is fuzzy pre-compact.

Proof:

Suppose that $\left\{\widetilde{N}_{\lambda}: \lambda \in \Lambda\right\}$ be a fuzzy pre- open cover of

Since $\mathrm{f}$ is fuzzy pre*- continuous and $\left\{\mu_{f^{-1}\left(\widetilde{N}_{\lambda}\right)}(\mathrm{x}): \lambda \in \boldsymbol{\Lambda}\right\}$ is a fuzzy pre- open set cover of $\mathrm{S}(\tilde{C})$ in $\tilde{A}$

Since $\tilde{C}$ is a fuzzy pre- open compact relative to $\tilde{A}$

There is a finite subfamily $\left\{\mu_{f^{-1}\left(\widetilde{N}_{\lambda}\right)}(\mathrm{x}): \boldsymbol{\lambda} \in \boldsymbol{\Lambda}\right\}$

Such that $\mu_{\mathrm{S}(\tilde{C})}(\mathrm{x}) \leq \max \left\{\mu_{f^{-1}\left(\widetilde{N}_{\lambda}\right)}(\mathrm{x})\right\}=f^{-1} \max \left\{\mu_{f^{-1}\left(\widetilde{N}_{\lambda}\right)}(\mathrm{x}): \lambda \in \boldsymbol{\Lambda}\right\}$

$\mu_{\mathrm{S}(f(\tilde{C})}(\mathrm{x})=\mu_{f(\mathrm{~S}(\tilde{C})}(\mathrm{x}) \leq \mathrm{f} f^{-1} \max \left\{\mu_{\left(\widetilde{N}_{\lambda}\right)}(\mathrm{x}): \lambda \in\right\} \leq \max \left\{\mu_{\left(\widetilde{N}_{\lambda}\right)}(\mathrm{x}): \lambda \in \boldsymbol{\Lambda}\right\}$

Therefore $\mathrm{f}(\tilde{C})$ is a fuzzy pre- compact relative to $\tilde{B}$

Propositions 3.12: [3]

1) If $\mathrm{f:}(\tilde{A}, \tilde{T}) \rightarrow\left(\tilde{B}, \tilde{T}^{\prime}\right)$ is a fuzzy pre*-open and bijective function and $\tilde{B}$ be fuzzy pre- compact then $\tilde{A}$ is a fuzzy pre- compact. 
Proof:

Suppose that $\left\{\widetilde{N}_{\lambda}: \lambda \in \Lambda\right\}$ be a family of a fuzzy pre- open covering of $\tilde{A}$

Let $\left\{\mu_{f\left(\widetilde{N}_{\lambda}\right)}(\mathrm{x}): \lambda \in \Lambda\right\}$ be a fuzzy pre-open set covering of $\widetilde{B}$

Since $\widetilde{B}$ is fuzzy pre- compact then there exist a finite family $\Lambda^{-} \subseteq \Lambda$ covers $\widetilde{B}$

Such that $\left\{\mu_{f\left(\widetilde{N}_{\lambda}\right)}(\mathrm{x}): \lambda \in \Lambda^{-}\right\}$covers $\tilde{B}$

Since $\mathrm{f}$ is bijective

Then $\mu_{f^{-1}(\tilde{B})}(\mathrm{x})=\mu_{(\tilde{A})}(\mathrm{x})=\mu_{f f^{-1}} \max \left\{\widetilde{N}_{\lambda}(\mathrm{x}): \lambda \in \Lambda\right\}=\max \left\{\widetilde{N}_{\lambda}(\mathrm{x}): \lambda \in \Lambda\right\}$

Hence $\tilde{A}$ is a fuzzy pre- compact

2) Let $\mathrm{f}:(\widetilde{\mathrm{A}}, \widetilde{\mathrm{T}}) \rightarrow\left(\widetilde{B}, \widetilde{T}^{\prime}\right)$ be a fuzzy pre- continuous surjective function and $\widetilde{\mathrm{A}}$ is a fuzzy pre- closed compact then $\widetilde{B}$ is a fuzzy pre- closed compact.

Proof:

Obvious

3) Let if $\mathrm{f}:(\widetilde{\mathrm{A}}, \widetilde{\mathrm{T}}) \rightarrow\left(\widetilde{B}, \widetilde{T}^{\prime}\right)$ be a fuzzy -pre continues surjective function of a fuzzy pre- compact a space $\widetilde{\mathrm{A}}$ onto a space $\widetilde{\mathrm{B}}$ then $\widetilde{\mathrm{B}}$ is fuzzy pre- compact.

Proof:

Obvious

4) Let if $\mathrm{f}:(\widetilde{\mathrm{A}}, \widetilde{\mathrm{T}}) \rightarrow\left(\widetilde{B}, \widetilde{T}^{\prime}\right)$ be a fuzzy -pre continues bijective function and $\widetilde{\mathrm{B}}$ be a fuzzy pre- compact space $\widetilde{\mathrm{A}}$ then $\widetilde{B}$ is fuzzy Pre- compact.

Proof

Obvious

Remark 3.13: [7] The following diagram explains the relationships among the different types of fuzzy continuous function.

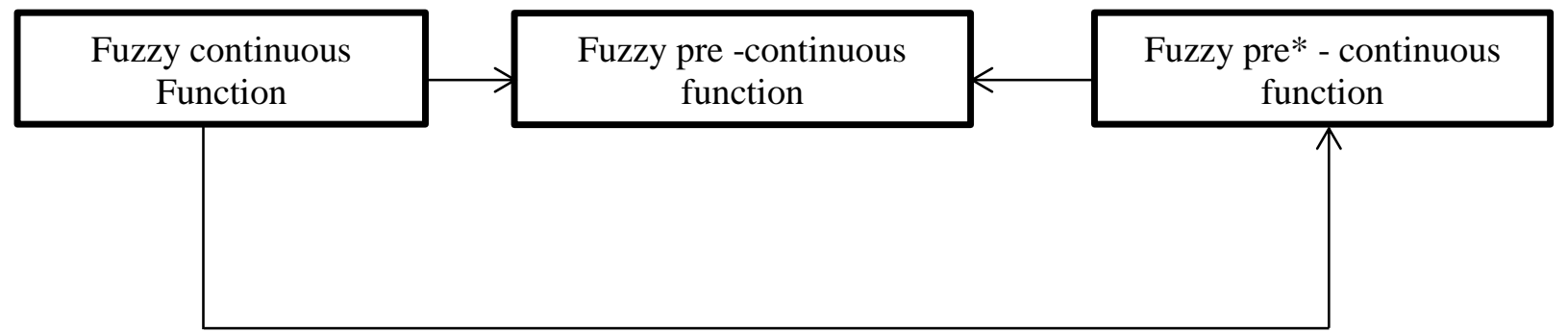

\section{Conclusion}

It is an interesting exercise to work on fuzzy, pre-continuous and fuzzy pre*-continuous; function of fuzzy pre-compact space in fuzzy topological space similarly other forms of fuzzy pre-open set can be applied to difine different forms of fuzzy pre-compact space.

Acknowledgements

The authors are grateful to the colleges' education department of mathematics. Al- Mustansiriya University, Baghdad, Iraq for its financial support.

\section{References}

[1] Chakraborty M.K. and T.M.G.Ahsanullah " Fuzzy topology on fuzzy sets and tolerance topology" fuzzy sets and systems, 45103-1o8 (1992)

[2] Chauldhuri M.K and P. Das" some results on fuzzy topology on fuzzy sets" fuzzy sets and systems, 56pp.331-336(1993).

[3] Ganesan Balasubramanian " on fuzzy $\beta$-compact and fuzzy $\beta$ - extermally disconnected space " vol.33 (1997), No 3,271-277

[4] Neeran Tahir AlKhafaji "on certain Types of-compact spaces " (2002)

[5] I.M.Hanafy " fuzzy $\beta$ - compactness and Fuzzy $\beta$ - closed spaces " 28(2004), 281-293

[6] Ahmed Ibrahem Nasir " Some Kinde Of strongly compact and pair- wise compact space" Baghdad University (2005)

[7] Suaad Gedaan Gasim " on semi -p-compact space " Baghdad University (2006)

[8] Saleem yaseen mageed "on fuzzy compact space " AL Mustansirya University (2012)

[9] V.Seenivasan, K, Kamala " fuzzy e-continuity and fuzzy e-open sets "volume x, No, x, (mm201y) pp.1-xx (2014) 